\title{
Maternal Dietary Patterns during Third Trimester in Association with Birthweight Characteristics and Early Infant Growth
}

\author{
Anna K. Poon, ${ }^{1,2}$ Edwina Yeung, ${ }^{1}$ Nansi Boghossian, ${ }^{1}$ Paul S. Albert, ${ }^{3}$ and Cuilin Zhang $^{1}$ \\ ${ }^{1}$ Epidemiology Branch, Division of Intramural Population Health Research, Eunice Kennedy Shriver National Institute of Child Health \\ and Human Development, 6100 Executive Boulevard, 7B03, Bethesda, MD 20892, USA \\ ${ }^{2}$ Department of Epidemiology, Johns Hopkins Bloomberg School of Public Health, 2024 E. Monument Street, \\ Baltimore, MD 21205, USA \\ ${ }^{3}$ Biostatistics and Bioinformatics Branch, Division of Intramural Population Health Research, Eunice Kennedy Shriver National \\ Institute of Child Health and Human Development, 6100 Executive Boulevard, 7B03, Bethesda, MD 20892, USA
}

Correspondence should be addressed to Edwina Yeung; yeungedw@mail.nih.gov

Received 20 November 2013; Accepted 15 December 2013

Academic Editors: I. Le Huërou-Luron, P. J. Schluter, and P. D. Terry

Copyright ( $) 2013$ Anna K. Poon et al. This is an open access article distributed under the Creative Commons Attribution License, which permits unrestricted use, distribution, and reproduction in any medium, provided the original work is properly cited.

\begin{abstract}
Our analysis examined the impact of maternal dietary patterns and lifestyle factors on markers of fetal growth, specifically birthweight and size for gestational age (small- (SGA) or large-for-gestational age (LGA)). The Infant Feeding Practices Study II, a prospective cohort study, surveyed pregnant women during their 3rd trimester, of which a subgroup $(n=893)$ completed a food frequency questionnaire. Maternal dietary patterns were evaluated by diet scores (Alternative Healthy Eating Index for Pregnancy and alternate Mediterranean diet) and by carbohydrate quality (glycemic index and glycemic load). Poisson regression with robust standard errors was used to examine the relative risk of SGA and separately LGA, with dietary patterns and other lifestyle factors. Linear regression was used to determine the association of birthweight and early infant growth with better dietary patterns. Relative risk of SGA and LGA was not associated with dietary patterns. Birthweight and infant growth were not associated with maternal diet. Smoking, however, increased the risk of delivering an SGA infant (RR = 2.92, 95\% CI: 1.58-5.39), while higher prepregnancy BMI increased the risk of delivering an LGA infant ( $\mathrm{RR}=1.06,95 \% \mathrm{CI}: 1.03-1.09)$. Future studies are needed to evaluate whether deficiencies in more specific maternal dietary nutrients play a role in fetal growth.
\end{abstract}

\section{Introduction}

Fetal growth is an important determinant not only of infant survival but also of future chronic disease risk. Both low and high birthweight have been associated with increased infant mortality and long-term morbidity $[1,2]$. Low birthweight has additionally been associated with elevated risk of type 2 diabetes [3], while high birthweight for gestational age has been associated with increased risk of overweight and obesity in adulthood $[4,5]$. Due to the lifelong implications of fetal growth defined by size-at-birth, further research is needed to understand its determinants.

Maternal nutrition is the major fuel for fetal growth [6]. While many studies have examined the role of individual nutrients during pregnancy [7-9], recent focus on nutritional epidemiology has shifted from examining the effect of single nutrients to assessing overall diet quality. Assessing nutrition as a dietary index may be more informative as it accounts for the combined effect of nutrients in foods [10]. In this regard, existing analyses on prenatal dietary patterns with birthweight have been scant and findings have been inconsistent [11]. As a result, further research is needed to understand the role of maternal dietary patterns in association with birthweight.

Our objective was to determine the association of overall maternal dietary patterns, as evaluated by the Alternative Healthy Eating Index for Pregnancy (AHEI-P) and the alternate Mediterranean diet (aMED), with birthweight, birthweight-for-gestational age (large and small), and early infant growth by 4-6 months of life in the Infant Feeding Practices Study II (IFPSII). Furthermore, the association of carbohydrate quality and quantity, as measured by the average glycemic index (GI) of diet and glycemic load (GL), was investigated as well. 


\section{Materials and Methods}

2.1. Study Population. IFPSII (2005-2007) is a longitudinal cohort study, sampling US women from a nationally distributed consumer opinion panel during their 3rd trimester of pregnancy [12]. After delivery, mothers and infants were eligible to be in the study if the infant was a healthy singleton delivered after at least 35 weeks of gestation, weighed at least 5 pounds, and did not stay in the intensive care unit for more than 3 days. Also, neither the mother nor the infant could have a medical condition that affected infant feeding. Longitudinal data were collected through mailed questionnaires from late pregnancy to 12 months postpartum. A subsample of 1,502 women completed and returned a food frequency questionnaire (FFQ) during the 3rd trimester of pregnancy. Of these, 1,032 remained in the study after the exclusions for the above criteria and other disqualifications [12]. Exclusions were also made for caloric intake in the top $2 \%$ or bottom $1 \%$ of energy intake (corresponding to women with caloric intake above $4,539 \mathrm{kcal}$ and below $606 \mathrm{kcal}$ ). The IFPSII study was approved by the FDA institutional Review Board.

2.2. Maternal Characteristics. Maternal demographics were either available via the panel database or collected through a short demographic questionnaire [12]. Examined demographic characteristics included: maternal age (years), maternal race (white or nonwhite), education (high school or less, some college, associate or bachelor, or master or more), and poverty index ratio $(<185 \%, 185$ to $350 \%$, or $\geq 350 \%)$. The poverty index ratio describes a family's income relative to their poverty threshold; poverty thresholds vary by family size. Index ratios $\leq 100 \%$ indicate that the family is living at or below the poverty level [13]. Prepregnancy weight $\left(\mathrm{kg} / \mathrm{m}^{2}\right)$ and smoking (yes or no) during pregnancy were reported in the prenatal questionnaire during the $3 \mathrm{rd}$ trimester. Maternal alcohol use $(\mathrm{g})$ and gestational weight gain $(\mathrm{kg})$ were reported in the prenatal FFQ and neonatal questionnaire, respectively.

2.3. Maternal Food Frequency Intake. The Diet History Questionnaire (DHQ) is a validated FFQ originally developed by the National Cancer Institute. The DHQ was modified to reflect dietary intake in the past month rather than the past year and included additional foods and nutrients relevant to pregnant women, such as specific types of fish and dietary supplements [12]. As a result, the modified DHQ mailed to women during their 3rd trimester reflects dietary intake between 28 and 36 weeks of gestation. The DHQ assessments were processed using the NCI's Diet ${ }^{*}$ Calc software (version 1.4.3), which produces nutrient, food category, and glycemic load estimates. The two dietary pattern indices calculated from the DHQ data include the Alternative Healthy Eating Index for Pregnancy (AHEI-P), our primary measure of overall dietary patterns, and the alternate Mediterranean diet (aMED). The average glycemic index (GI) and glycemic load (GL) were also derived from DHQ data as measures of carbohydrate quality.

2.4. Alternative Healthy Eating Index for Pregnancy (AHEI$P$ ). The original Healthy Eating Index (HEI) was developed to measure overall dietary patterns based on the 1995 Food Guide Pyramid and Dietary Guidelines for Americans [14]. Since then, the HEI has been modified periodically to reflect changes in dietary recommendations. Our AHEI$\mathrm{P}$ is based on a 130-point scale with $0-10$ points awarded for optimal intake of 13 types of foods and nutrients. The score was adapted from the recently updated AHEI-2010 by Chiuve et al. and an earlier pregnancy AHEI score by RifasShiman et al. $[15,16]$. To make the AHEI-2010 suitable for dietary assessment in pregnant women, alcohol was excluded, while calcium, folate, and iron were added to the scoring method [16]. Participants received higher scores for higher intakes of healthier components consisting of vegetables, whole fruit, whole grains, nuts and legumes, long-chain (n-3) fats, polyunsaturated fats, folate, calcium, and iron. Higher intakes of less healthy components including sugarsweetened beverages, red and processed meat, trans fat, and sodium received lower scores. For healthier components, the mother's observed intake was divided by the criterion for maximum points and multiplied by 10 . To assign higher points for lower intake of less healthy components, the mother's observed intake was divided by the criterion for maximum points, subtracted from 1 , and then multiplied by 10. Sodium intake was divided into eleven groups and scored on a $0-10$ point scale. All of the above scores for the individual dietary components were then summed to get the total AHEI$\mathrm{P}$ score for each mother with higher scores indicating better dietary patterns. AHEI-P was categorized into tertiles and also examined as a continuous variable.

2.5. Alternate Mediterranean Diet (aMED). The traditional Mediterranean diet, originally derived from dietary intake observed in the Mediterranean region, distinguishes itself from the AHEI-P based on its different nutrient requirements, such as low intake of saturated fats [17]. To measure adherence to the Mediterranean diet in pregnant women, the score was modified to exclude alcohol intake. Based on an 8-point scale, participants received 1 point if their intake of healthier components exceeded the median intake and if their intake of less healthy components was below the median. The median value for each component was based on the distribution of intake within IFPSII. Healthier components included vegetables, legumes, fruits, nuts, whole grains, fish, and the ratio of monounsaturated to saturated fats, while less healthy components consisted of red and processed meats. The points for each category were summed to get the total aMED for each mother with a higher score indicating better adherence. aMED was categorized into low (0-3), moderate (4-5), and high (6-8) adherence groups and also examined as a continuous variable.

2.6. Glycemic Index (GI) and Glycemic Load (GL). GI is a measure of the glycemic effect of a particular food relative to a standard amount of glucose. GL is a measure of both the quality and quantity of carbohydrate consumption and is calculated based on a food's GI [18]. Both GI and GL differ from the AHEI-P and aMED because they solely capture responses to carbohydrates in diet. GL was estimated from each participant's FFQ response using the Diet ${ }^{*}$ Calc software. 
Overall average GI of a participant's diet was calculated by dividing GL by total carbohydrate intake and multiplying it by 100 [18]. Both GI and GL were categorized into tertiles and examined as continuous variables in analysis.

2.7. Size-at-Birth and Infant Growth. Infant's birthweight was reported on the birth screener after delivery. Gestational age was determined based on the mother's expected date of delivery and the infant's birth date, as reported on the birth screener. Infant's birth length and gender were reported on the neonatal questionnaire. Data on gender, birthweight, and gestational age were used to identify infants as small-forgestational age (SGA, defined as gender-specific birthweightfor-gestational age $\leq 10$ th percentile) or large-for-gestational age (LGA, defined as gender-specific birthweight-for-gestational age $\geq 90$ th percentile) [19]. Birthweight $\mathrm{z}$-scores and weight-for-length (WFL) z-scores at birth were additionally calculated based on 2000 CDC reference growth charts.

At the 3rd-, 5th-, 7th-, and 12th-month postpartum mailed surveys, mothers reported their infant's weight and length and the date of each measurement based on their last pediatrician's visit. From these questionnaires, weight and length measurements were used to calculate WFL z-scores when the infant's age was between 4 and 6 months using the 2000 CDC growth charts. This age was chosen because change in WFL in the first 6 months of life is a marker for accelerated infant growth.

2.8. Statistical Analysis. The final analytic sample included 893 participants after exclusions for gestational ages above 43.5 weeks $(n=6)$, type I or type II diabetes $(n=9)$, or missing gender information $(n=66)$. Associations between tertiles of AHEI-P and maternal covariates were assessed using ANOVA for continuous variables and $\chi^{2}$-test for categorical variables. Poisson regression with robust standard errors [20] was used to estimate the relative risks and 95\% confidence intervals for the associations of SGA and LGA by AHEI-P tertiles, adjusting for total energy intake, maternal age, race, education, poverty index ratio, maternal prepregnancy BMI, and smoking and alcohol consumption during pregnancy. This analysis was repeated with the aMED categories, GI tertiles, and GL tertiles.

Multiple linear regression analysis was used to determine the association of birthweight, WFL z-score at birth, and WFL z-score at 4-6 months with AHEI-P, aMED, GI, and GL. Models for birthweight and birth WFL $\mathrm{z}$-score were fully adjusted for the above-mentioned covariates with additional adjustment for gestational age. Change in infant growth as an outcome was modeled with WFL z-score at 4-6 months, adjusting for gestational age, WFL $\mathrm{z}$-score at birth, and all the other covariates [16]. All associations are presented with their respective estimates and 95\% confidence intervals with statistical significance at $P<0.05$. Data analyses were performed using SAS 9.3 (SAS Institute Inc., Cary, NC, USA).

\section{Results}

3.1. Baseline Characteristics. The mean ( \pm standard deviation) AHEI-P, aMED, GI, and GL values were $59.1 \pm 11.8$ points, 4.0 \pm 1.8 points, $50.0 \pm 3.6$ percent, and $141.5 \pm 66.0$ g, respectively. Total food energy increased with higher AHEI-P tertiles $(P<$ $0.0001)$. Women with higher AHEI-P scores tended to be older, with higher education, lower poverty index ratio, and lower prepregnancy BMI (Tables 1 and 2). Women excluded from the study sample did not have significantly different mean scores of prenatal dietary indices from those included in the study (data not shown).

3.2. Association of AHEI-P with Size-for-Gestational Age. Eighty-two (9.2\%) infants were LGA. No significant associations between AHEI-P tertiles and LGA were observed. The relative risk of the highest versus the lowest tertile of AHEI$\mathrm{P}$ was 0.92 (95\% confidence interval (CI): 0.50-1.69). LGA, however, was associated with prepregnancy BMI (Relative Risk (RR) $=1.06,95 \%$ CI: 1.03-1.09). aMED, GI, and GL were not associated with LGA (Table 3 ).

Seventy-one (8.0\%) infants were SGA. Similarly, after adjusting for covariates, no significant differences were examined across the tertiles of AHEI-P in association with SGA risk. The risk estimate of the highest tertile compared to the lowest tertile of AHEI-P was 0.93 (95\% CI: 0.49-1.75). SGA, however, was associated with maternal race $(\mathrm{RR}=3.16$, 95\% CI: 1.97-5.06) and smoking status $(\mathrm{RR}=2.92,95 \% \mathrm{CI}$ : 1.58-5.39). aMED, GI, and GL were not associated with SGA (Table 3). Stratifying by smoking status did not alter our findings with AHEI-P and neither did excluding women with gestational diabetes $(n=46)$ (data not shown).

3.3. Association of AHEI-P with Birthweight, Birth WFL, and Change in WFL $z$-Scores. Neither birthweight z-scores nor birth WFL z-scores were associated with AHEI-P scores (Table 4). The mean differences in $\mathrm{Z}$-score units per unit increase in AHEI-P were 0.002 (95\% CI: -0.003-0.008) and 0.005 (95\% CI: -0.004-0.013), respectively. AHEI-P was also not associated with change in WFL at 4-6 months (difference $=0.009,95 \%$ CI: $-0.004-0.023$ ). Fully adjusted models with aMED, GI, and GL were similarly not associated with birthweight, birth WFL, and change in WFL z-scores at 4-6 months.

\section{Discussion}

In a nationally distributed sample of 893 US mothers of healthy singletons delivered after 35 weeks of gestation, maternal dietary patterns and carbohydrate quality during third trimester were not associated with offspring outcomes consisting of birthweight, size-for-gestational age (small or large), and infant growth in the first 4-6 months of life. On the other hand, maternal characteristics including race, smoking status, and prepregnancy BMI were associated with SGA and LGA risk.

Two previous studies have examined dietary patterns as measured by AHEI-P in association with birthweight outcomes. In the Infancia y Medio Ambiente (INMA) cohort of 787 pregnant Spanish women, investigators found a reduced risk of fetal growth restriction (FGR) for weight (defined as infants below the lower limit of the $80 \%$ CI for predicted birthweight based on maternal and paternal anthropometry) 
TABLE 1: Alternative Healthy Eating Index for Pregnancy (AHEI-P) a scoring method and mean AHEI-P scores for 893 women in the Infant Feeding Practices Study II.

\begin{tabular}{lccc}
\hline Component & Criterion for minimum (0) & Criterion for maximum (10) & Mean score \pm SD \\
\hline Vegetables, servings/d & 0 & $\geq 5$ & $5.0 \pm 2.7$ \\
Whole fruit, servings/d & 0 & $\geq 4$ & $4.4 \pm 3.1$ \\
Whole grains, g/d & 0 & 0 & $2.5 \pm 1.5$ \\
Sugar-sweetened beverages, servings/d & $\geq 1$ & $\geq 1$ & $2.0 \pm 3.3$ \\
Nuts and legumes, servings/d & 0 & 0 & $3.7 \pm 2.8$ \\
Red/processed meat, servings/d & $\geq 1.5$ & $\leq 0.5$ & $1.4 \pm 2.3$ \\
trans Fat, \% of energy & $\geq 4$ & 250 & $5.5 \pm 1.6$ \\
Long-chain $(n=3$ ) fats (EPA + DHA), mg/d & 0 & $\geq 10$ & $2.5 \pm 2.2$ \\
PUFA, \% of energy & $\leq 2$ & Lowest decile & $5.6 \pm 2.1$ \\
Sodium, mg/d & Highest decile & $\geq 1200$ & $5.2 \pm 3.1$ \\
Calcium, mg/d & 0 & $\geq 600$ & $8.3 \pm 2.2$ \\
Folate, mcg/d & 0 & $\geq 27$ & $6.9 \pm 2.2$ \\
Iron, mg/d & 0 & - & $6.0 \pm 2.1$ \\
AHEI-P & - & $59.1 \pm 11.8$ \\
\hline
\end{tabular}

${ }^{\mathrm{a}}$ The AHEI-P score was adapted from Rifas-Shiman et al. 2009 and Chiuve et al. 2012 [15, 16]; out of 130 points.

TABLE 2: Baseline characteristics of maternal covariates by tertiles of AHEI-P.

\begin{tabular}{|c|c|c|c|c|c|}
\hline & $\mathrm{T} 1$ & $\mathrm{~T} 2$ & $\mathrm{~T} 3$ & $P$-value & Overall \\
\hline \multicolumn{6}{|l|}{ Maternal demographics } \\
\hline Age, years & $28.4 \pm 5.3$ & $28.7 \pm 5.1$ & $30.1 \pm 5.5$ & $<0.001$ & $29.1 \pm 5.4$ \\
\hline \multicolumn{6}{|l|}{ Race, $n(\%)$} \\
\hline White & $264(89.5)$ & $261(87.9)$ & $251(84.8)$ & 0.22 & $777(87.4)$ \\
\hline \multicolumn{6}{|l|}{ Education, $n(\%)$} \\
\hline High school or less & $68(23.9)$ & $42(14.7)$ & $43(15.3)$ & $<0.001$ & $154(18.0)$ \\
\hline Some College & $126(44.2)$ & $108(37.8)$ & $102(36.2)$ & & $336(39.3)$ \\
\hline Associate or BA & $73(25.6)$ & $97(33.9)$ & $101(35.8)$ & & $271(31.7)$ \\
\hline Master or more & $18(6.3)$ & $39(13.6)$ & $36(12.8)$ & & $93(10.9)$ \\
\hline \multicolumn{6}{|l|}{ Poverty index ratio, $n(\%)$} \\
\hline$<185 \%$ & $138(46.5)$ & $105(35.2)$ & $92(31.0)$ & $<0.0001$ & $335(37.5)$ \\
\hline 185 to $350 \%$ & $114(38.4)$ & $118(39.6)$ & $113(38.1)$ & & $346(38.8)$ \\
\hline$\geq 350 \%$ & $45(15.2)$ & $75(25.2)$ & $92(31.0)$ & & $212(23.7)$ \\
\hline \multicolumn{6}{|l|}{ Maternal health } \\
\hline Smoked, $n(\%)$ & $31(10.5)$ & $25(8.5)$ & $17(5.8)$ & 0.11 & $74(8.3)$ \\
\hline Alcohol, g & $0.1 \pm 0.3$ & $0.1 \pm 0.2$ & $0.2 \pm 1.5$ & 0.16 & $0.1 \pm 0.9$ \\
\hline Prepregnancy BMI, kg/m² & $27.5 \pm 7.3$ & $25.5 \pm 5.8$ & $25.3 \pm 5.7$ & $<0.0001$ & $26.1 \pm 6.4$ \\
\hline Gestational weight gain, $\mathrm{kg}$ & $13.4 \pm 6.4$ & $14 \pm 6.1$ & $14.4 \pm 5.8$ & 0.12 & $14.0 \pm 6.1$ \\
\hline Total food energy, kcal & $1733 \pm 660$ & $2036 \pm 729$ & $2546 \pm 969$ & $<0.0001$ & $2104 \pm 864$ \\
\hline \multicolumn{6}{|l|}{ Infant Health } \\
\hline Gestational age, weeks & $39.3 \pm 1.2$ & $39.3 \pm 1.3$ & $39.3 \pm 1.2$ & 0.62 & $39.3 \pm 1.2$ \\
\hline \multicolumn{6}{|l|}{ Birthweight } \\
\hline Birthweight, g & $3444 \pm 446$ & $3405 \pm 462$ & $3478 \pm 454$ & 0.14 & $3443 \pm 454$ \\
\hline BW $\leq 10$ th percentile, $n(\%)$ & $24(8.1)$ & $23(7.7)$ & $24(8.1)$ & 0.98 & $71(7.95)$ \\
\hline BW $\geq 90$ th percentile, $n(\%)$ & $32(10.8)$ & $22(7.4)$ & $28(9.4)$ & 0.35 & $82(9.18)$ \\
\hline \multicolumn{6}{|l|}{ Diet scores } \\
\hline AHEI-P, range $(n)$ & $33-52(297)$ & $53-62(298)$ & $63-98(297)$ & & $59.1 \pm 11.8$ \\
\hline $\mathrm{aMED}^{\mathrm{a}}$, range $(n)$ & $0-3(369)$ & 4-5 (333) & $6-8(191)$ & & $4.0 \pm 1.8$ \\
\hline \multicolumn{6}{|l|}{ Carbohydrate quality } \\
\hline $\mathrm{GI}^{\mathrm{b}}$, range $(n)$ & $35-48(297)$ & $49-51(298)$ & $52-63(298)$ & & $50.0 \pm 3.6$ \\
\hline $\mathrm{GL}^{\mathrm{c}}$, range $(n)$ & $38-107(297)$ & $108-152(298)$ & $153-520(298)$ & & $141.5 \pm 66.0$ \\
\hline
\end{tabular}

${ }^{\mathrm{a}}$ Alternate Mediterranean diet, out of 8 pts; ${ }^{\mathrm{b}}$ glycemic index; ${ }^{\mathrm{c}}$ glycemic load. 
TABLE 3: Risk ratios and 95\% confidence intervals for the association of SGA and LGA with dietary pattern indices.

\begin{tabular}{|c|c|c|}
\hline & $\begin{array}{c}\text { SGA }(n=71) \\
\text { Estimate }(95 \% \mathrm{CI})\end{array}$ & $\begin{array}{c}\text { LGA }(n=82) \\
\text { Estimate }(95 \% \mathrm{CI})\end{array}$ \\
\hline \multicolumn{3}{|l|}{ Model with AHEI-P } \\
\hline$n$ (number of obs. used) & 755 & 775 \\
\hline \multicolumn{3}{|l|}{ AHEI-P } \\
\hline T2 (53-62) versus T1 (33-52) & $0.73(0.41,1.31)$ & $0.74(0.43,1.26)$ \\
\hline T3 (63-98) versus T1 (33-52) & $0.93(0.49,1.75)$ & $0.92(0.50,1.69)$ \\
\hline Maternal age & $0.95(0.90,1.01)$ & $0.99(0.96,1.03)$ \\
\hline Nonwhite versus White & $3.16(1.97,5.06)$ & $0.43(0.16,1.15)$ \\
\hline \multicolumn{3}{|l|}{ Education } \\
\hline Some college versus $\leq \mathrm{HS}$ & $1.29(0.68,2.43)$ & $1.15(0.61,2.20)$ \\
\hline Associate or BA versus $\leq \mathrm{HS}$ & $0.71(0.30,1.68)$ & $1.62(0.81,3.23)$ \\
\hline Master or more versus $\leq \mathrm{HS}$ & $1.03(0.35,3.08)$ & $1.39(0.57,3.37)$ \\
\hline \multicolumn{3}{|l|}{ Poverty index ratio } \\
\hline (185 to $350 \%)$ versus $(<185 \%)$ & $1.16(0.63,2.12)$ & $1.18(0.70,1.97)$ \\
\hline$(\geq 350 \%)$ versus $(<185 \%)$ & $1.83(0.91,3.65)$ & $0.89(0.45,1.73)$ \\
\hline Smoked, yes versus no & $2.92(1.58,5.39)$ & $0.76(0.29,1.97)$ \\
\hline Alcohol, g & $0.97(0.82,1.15)$ & $0.47(0.10,2.13)$ \\
\hline Total energy & $1.00(1.00,1.00)$ & $1.00(1.00,1.00)$ \\
\hline Prepregnancy BMI, kg/m² & $0.98(0.94,1.02)$ & $1.06(1.03,1.09)$ \\
\hline \multicolumn{3}{|l|}{ Model with aMED ${ }^{\mathrm{a}}$} \\
\hline T2 (4-5) versus T1 (0-3) & $0.75(0.44,1.29)$ & $0.71(0.44,1.14)$ \\
\hline T3 (6-8) versus T1 (0-3) & $0.94(0.48,1.81)$ & $0.71(0.37,1.35)$ \\
\hline \multicolumn{3}{|l|}{ Model with $G I^{\mathrm{a}}$} \\
\hline T1 (35-48) versus T3 (52-63) & $0.86(0.49,1.49)$ & $1.03(0.60,1.75)$ \\
\hline T2 (49-51) versus T3 (52-63) & $0.87(0.50,1.50)$ & $1.26(0.77,2.08)$ \\
\hline \multicolumn{3}{|l|}{ Model with $G L^{\mathrm{a}}$} \\
\hline T1 (38-107) versus T3 (153-520) & $0.92(0.35,2.40)$ & $1.18(0.50,2.78)$ \\
\hline T2 (108-152) versus T3 $(153-520)$ & $1.13(0.53,2.37)$ & $1.10(0.56,2.17)$ \\
\hline
\end{tabular}

${ }^{\text {a }}$ Adjusted for total energy intake, race, education, age, poverty index ratio, smoking, alcohol, and pre-pregnancy BMI.

TABLE 4: Mean differences in birth weight, weight-for-length (WFL), and change in WFL z-scores per unit increase in dietary pattern indices.

\begin{tabular}{lccc}
\hline & $\begin{array}{c}\text { Birthweight }^{\mathrm{a}} \\
\text { Estimate }(95 \% \mathrm{CI})\end{array}$ & $\begin{array}{c}\text { Birth WFL }^{\mathrm{a}} \\
\text { Estimate }(95 \% \mathrm{CI})\end{array}$ & $\begin{array}{c}\text { Change in WFL at 4-6 months } \\
\text { Estimate }(95 \% \text { CI })\end{array}$ \\
\hline$n$ (number of obs. used) & 815 & 815 & 426 \\
AHEI-P & $0.002(-0.003,0.008)$ & $0.005(-0.004,0.013)$ & $0.009(-0.004,0.023)$ \\
aMED & $-0.003(-0.036,0.031)$ & $0.03(-0.03,0.08)$ & $0.06(-0.03,0.14)$ \\
GI & $-0.015(-0.031,0.001)$ & $-0.02(-0.04,0.01)$ & $-0.01(-0.04,0.03)$ \\
GL & $-0.002(-0.004,0.001)$ & $0.0004(-0.0028,0.0036)$ & $-0.003(-0.009,0.002)$ \\
\hline
\end{tabular}

${ }^{a}$ Adjusted for total energy intake, race, education, age, poverty index ratio, smoking, alcohol, pre-pregnancy BMI, and gestational age; ${ }^{\mathrm{b}}$ also adjusted for birth WFL.

per five-point increase in AHEI-P (Odds Ratio $(\mathrm{OR})=0.70$, 95\% CI: 0.59-0.85) [21]. Better dietary patterns also predicted higher birthweight $(P$ trend $=0.009)[21]$. However, findings from the US, including the results of our analysis indicating no association, have not been as supportive of such an effect. In Project Viva, a prospective cohort of 1,777 pregnant US women, a nonsignificant lowering of risk of both SGA (OR $=0.92,95 \%$ CI: $0.82-1.02)$ and LGA (OR $=0.95,95 \%$ CI: 0.89-1.02) was identified per five-point increase in AHEI-P [16]. For comparison, reproducing the analysis in IFPSII with logistic regression and AHEI-P (per 5-point increment) did not alter the nonsignificant association with either SGA ( $\mathrm{OR}=0.98,95 \%$ CI: $0.86-1.12)$ or LGA $(\mathrm{OR}=0.99,95 \% \mathrm{CI}$ : $0.88-1.12$ ). As for measuring dietary patterns by the aMED, the INMA-Mediterranean cohort found lower risk ( $R R=$ 0.50, 95\% CI: 0.28-0.90) of delivering an infant with FGR for weight (defined as infants below the 10th percentile of the predicted birthweight distribution). The results, however, were not consistently protective in the INMA-Atlantic (RR $=0.97,95 \%$ CI: $0.42-2.26)$ or RHEA cohorts $(\mathrm{RR}=1.96,95 \%$ 
CI: 0.90-4.25) [22]. Also, a recent systematic review of eight studies examining low GI and GL diets suggests that further research is needed before recommending either pattern during pregnancy [23]. These findings taken together with our current analysis from IFPSII suggest that there remains a lack of evidence that prenatal dietary patterns during third trimester, as measured by the AHEI-P and aMED, and carbohydrate quality, based on GI and GL, can prevent SGA or LGA.

Our observation that prepregnancy weight affects birthweight suggests that diet is not completely irrelevant. As prepregnancy BMI is associated with infant size-at-birth, this association suggests prepregnancy diet may be related to fetal growth. In our study, mothers had a $6 \%$ higher risk (95\% CI: 1.03-1.09) of delivering an LGA infant per unit increase in BMI. These findings are consistent with previous studies [24-28]. Djelantik et al., for example, reported that overweight $(\mathrm{RR}=1.55,95 \% \mathrm{CI}: 1.30-1.84)$ and obese $(\mathrm{RR}=$ 2.03, 95\% CI: 1.60-2.59) women were significantly more likely to deliver an LGA infant compared to women with normal prepregnancy BMI [26]. Other authors additionally reported a positive association of underweight prepregnancy BMI (BMI $\left.<18.5 \mathrm{~kg} / \mathrm{m}^{2}\right)$ with intrauterine growth restriction compared to women with normal prepregnancy BMI $(18.5 \leq$ BMI < 25) [29]. These findings thus emphasize the importance of maternal prepregnancy weight as an early indicator of identifying women who may be at risk of delivering infants with abnormal birthweight.

Among pregnant US women with sufficient energy intake, smoking during pregnancy increased SGA risk. This result indicates that growth in utero depends on maternal factors other than prenatal dietary patterns. Smoking is a well-known modifiable risk factor for restricting fetal growth and our findings for smoking are in agreement with previous studies [30,31]. Our findings show that mothers who reported smoking during pregnancy were about three times (95\% CI: 1.58-5.39) more likely to deliver an SGA infant. Similarly, another study reported that mothers who smoked during pregnancy delivered infants who were $142 \mathrm{~g}$ lighter on average and two times (RR $=2.07,95 \% \mathrm{CI}: 1.69-2.53)$ more likely to be growth restricted than nonsmoking mothers [31]. These results are evidence of the adverse effects of maternal smoking habits on fetal growth and add to the mounting evidence of the importance of smoking cessation.

Our analysis should be interpreted in the context of the strengths and limitations of the study. Two key strengths include the prospective study design with the assessment of prenatal diet prior to the examined birth outcomes and the evaluation of maternal diet using validated measures of dietary patterns. There were also several limitations. First, while prenatal diet was only measured once during the third trimester, repeated measurements may have better captured dietary intake. Dietary intake in both the INMA and Project Viva cohorts reflected intake in the first trimester. However, we do not expect maternal diet to differ significantly between early and late pregnancy [32]. As such, differences in our findings in comparison to these cohorts are unlikely to be due to the exposure window. Second, gestational age was self-reported. Third, our sample size may have limited the statistical power of detecting a significant association. However, our sample size $(n=893)$ was comparable to the INMA cohort $(n=787)$. Fourth, the IFPSII population largely consisted of healthy singletons, which restricts the generalizability of our results.

Our findings suggest that prenatal dietary patterns as measured by AHEI-P and aMED, and carbohydrate quality based on GI and GL, during the 3rd trimester do not affect birth outcomes defined by birthweight, SGA and LGA, as well as infant growth at 4-6 months. Our results continue to emphasize the importance of prepregnancy BMI and smoking habits as key risk factors for SGA and LGA and as such continue to be clinically useful indicators for identifying women at risk for poor birth outcomes.

\section{Conflict of Interests}

The authors declare that they have no conflict of interests.

\section{Authors' Contribution}

Anna Poon, Edwina Yeung, and Nansi Boghossian conceived and designed the data analysis, carried out substantial parts of the data analysis, and interpreted the results. Anna Poon drafted the paper. Paul Albert and Cuilin Zhang contributed to the interpretation of the data. All authors contributed in revising the paper for important intellectual content.

\section{Acknowledgments}

Anna Poon, Edwina Yeung, Nansi Boghossian, Paul Albert, and Cuilin Zhang were supported by the Intramural Research Program of the Eunice Kennedy Shriver National Institute of Child Health \& Human Development, National Institutes of Health. IFPSII was funded by the Food and Drug Administration, Centers for Disease Control and Prevention, Office of Women's Health, National Institutes of Health, and Maternal and Child Health Bureau in the U.S. Department of Health and Human Services.

\section{References}

[1] D. D. Mcintire, S. L. Bloom, B. M. Casey, and K. J. Leveno, "Birth weight in relation to morbidity and mortality among newborn infants," The New England Journal of Medicine, vol. 340, no. 16, pp. 1234-1238, 1999.

[2] X. Zhang, A. Decker, R. W. Platt, and M. S. Kramer, "How big is too big? The perinatal consequences of fetal macrosomia," American Journal of Obstetrics and Gynecology, vol. 198, no. 5, pp. 517.e1-517.e6, 2008.

[3] J.-N. Wei, F.-C. Sung, C.-Y. Li et al., "Low birth weight and high birth weight infants are both at an increased risk to have type 2 diabetes among schoolchildren in Taiwan," Diabetes Care, vol. 26, no. 2, pp. 343-348, 2003.

[4] F. Ahlsson, J. Gustafsson, T. Tuvemo, and M. Lundgren, "Females born large for gestational age have a doubled risk of giving birth to large for gestational age infants," Acta Paediatrica, International Journal of Paediatrics, vol. 96, no. 3, pp. 358-362, 2007. 
[5] S. Cnattingius, E. Villamor, Y. T. Lagerros, A.-K. Wikström, and F. Granath, "High birth weight and obesity-a vicious circle across generations," International Journal of Obesity, vol. 36, pp. 1320-1324, 2012.

[6] I. Cetin, C. Berti, and S. Calabrese, "Role of micronutrients in the periconceptional period," Human Reproduction Update, vol. 16, no. 1, pp. 80-95, 2009.

[7] K. Godfrey, S. Robinson, D. J. P. Barker, C. Osmond, and V. Cox, "Maternal nutrition in early and late pregnancy in relation to placental and fetal growth," British Medical Journal, vol. 312, no. 7028, pp. 410-414, 1996.

[8] T. O. Scholl, M. L. Hediger, J. I. Schall, C.-S. Khoo, and R. L. Fischer, "Dietary and serum folate: their influence on the outcome of pregnancy," American Journal of Clinical Nutrition, vol. 63, pp. 520-525, 1996.

[9] G. Wu, F. W. Bazer, T. A. Cudd, C. J. Meininger, and T. E. Spencer, "Maternal nutrition and fetal development," Journal of Nutrition, vol. 134, no. 9, pp. 2169-2172, 2004.

[10] F. B. Hu, "Dietary pattern analysis: a new direction in nutritional epidemiology," Current Opinion in Lipidology, vol. 13, pp. 3-9, 2002.

[11] A. Sánchez-Villegas, N. Brito, J. Doreste-Alonso et al., "Methodological aspects of the study of dietary patterns during pregnancy and maternal and infant health outcomes. A systematic review," Maternal and Child Nutrition, vol. 6, supplement s2, pp. 100-111, 2010.

[12] S. B. Fein, J. Labiner-Wolfe, K. R. Shealy, R. Li, J. Chen, and L. M. Grummer-Strawn, "Infant feeding practices study II: study methods," Pediatrics, vol. 122, no. 2, pp. S28-S35, 2008.

[13] United States Census Bureau Poverty, 2013, http://www.census .gov/hhes/www/poverty/methods/definitions.html.

[14] E. T. Kennedy, J. Ohls, S. Carlson, and K. Fleming, "The healthy eating index: design and applications," Journal of the American Dietetic Association, vol. 95, no. 10, pp. 1103-1108, 1995.

[15] S. E. Chiuve, T. T. Fung, E. B. Rimm et al., "Alternative dietary indices both strongly predict risk of chronic disease," Journal of Nutrition, vol. 142, pp. 1009-1018, 2012.

[16] S. L. Rifas-Shiman, J. W. Rich-Edwards, K. P. Kleinman, E. Oken, and M. W. Gillman, "Dietary quality during pregnancy varies by maternal characteristics in project viva: A US cohort," Journal of the American Dietetic Association, vol. 109, no. 6, pp. 1004-1011, 2009.

[17] A. Trichopoulou, T. Costacou, C. Bamia, and D. Trichopoulos, "Adherence to a Mediterranean diet and survival in a Greek population," The New England Journal of Medicine, vol. 348, no. 26, pp. 2599-2608, 2003.

[18] C. A. McGowan and F. M. McAuliffe, "The influence of maternal glycaemia and dietary glycaemic index on pregnancy outcome in healthy mothers," British Journal of Nutrition, vol. 104, no. 2, pp. 153-159, 2010.

[19] M. S. Kramer, R. W. Platt, S. W. Wen et al., "A new and improved population-based Canadian reference for birth weight for gestational age," Pediatrics, vol. 108, no. 2, article E35, 2001.

[20] G. Zou, "A modified poisson regression approach to prospective studies with binary data," American Journal of Epidemiology, vol. 159, no. 7, pp. 702-706, 2004.

[21] C. L. Rodríguez-Bernal, M. Rebagliato, C. Iñiguez et al., "Diet quality in early pregnancy and its effects on fetal growth outcomes: The infancia y medio ambiente (childhood and environment) mother and child cohort study in Spain," The American Journal of Clinical Nutrition, vol. 91, no. 6, pp. 16591666, 2010.
[22] L. Chatzi, M. Mendez, R. Garcia et al., "Mediterranean diet adherence during pregnancy and fetal growth: INMA (Spain) and RHEA (Greece) mother-child cohort studies," British Journal of Nutrition, vol. 107, no. 1, pp. 135-145, 2012.

[23] J. C. Louie, J. C. Brand-Miller, T. P. Markovic, G. P. Ross, and R. G. Moses, "Glycemic index and pregnancy: a systematic literature review," Journal of Nutrition and Metabolism, vol. 2010, Article ID 282464, 8 pages, 2010.

[24] P. D. Padilha, E. Accioly, C. Chagas, E. Portela, C. L. Da Silva, and C. Saunders, "Birth weight variation according to maternal characteristics and gestational weight gain in Brazilian women," Nutricion Hospitalaria, vol. 24, no. 2, pp. 207-212, 2009.

[25] M. I. Cedergren, "Maternal morbid obesity and the risk of adverse pregnancy outcome," Obstetrics and Gynecology, vol. 103, no. 2, pp. 219-224, 2004.

[26] A. A. A. M. J. Djelantik, A. E. Kunst, M. F. Van Der Wal, H. A. Smit, and T. G. M. Vrijkotte, "Contribution of overweight and obesity to the occurrence of adverse pregnancy outcomes in a multi-ethnic cohort: population attributive fractions for Amsterdam," BJOG, vol. 119, no. 3, pp. 283-290, 2012.

[27] I. O. Frederick, M. A. Williams, A. E. Sales, D. P. Martin, and M. Killien, "Pre-pregnancy body mass index, gestational weight gain, and other maternal characteristics in relation to infant birth weight," Maternal and Child Health Journal, vol. 12, no. 5, pp. 557-567, 2008.

[28] A. S. Khashan and L. C. Kenny, "The effects of maternal body mass index on pregnancy outcome," European Journal of Epidemiology, vol. 24, no. 11, pp. 697-705, 2009.

[29] D. A. Doherty, E. F. Magann, J. Francis, J. C. Morrison, and J. P. Newnham, "Pre-pregnancy body mass index and pregnancy outcomes," International Journal of Gynecology and Obstetrics, vol. 95, no. 3, pp. 242-247, 2006.

[30] O. G. Brooke, H. R. Anderson, J. M. Bland, J. L. Peacock, and C. M. Stewart, "Effects on birth weight of smoking, alcohol, caffeine, socioeconomic factors, and psychosocial stress," British Medical Journal, vol. 298, no. 6676, pp. 795-801, 1989.

[31] B. L. Horta, C. G. Victora, A. M. Menezes, R. Halpern, and F. C. Barros, "Low birthweight, preterm births and intrauterine growth retardation in relation to maternal smoking," Paediatric and Perinatal Epidemiology, vol. 11, no. 2, pp. 140-151, 1997.

[32] N. Talai Rad, C. Ritterath, T. Siegmund et al., "Longitudinal analysis of changes in energy intake and macronutrient composition during pregnancy and 6 weeks post-partum," Archives of Gynecology and Obstetrics, vol. 283, no. 2, pp. 185-190, 2011. 


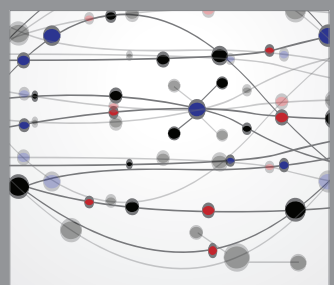

The Scientific World Journal
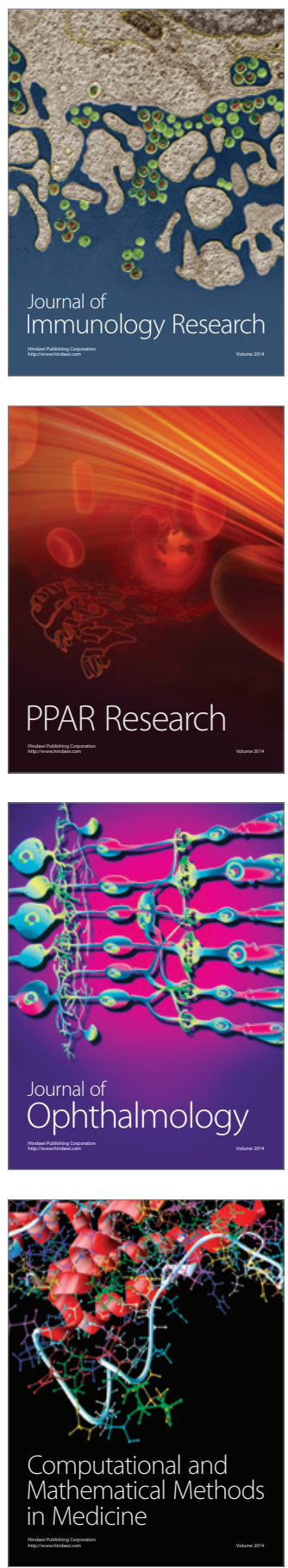

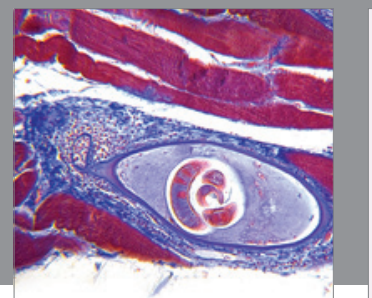

Gastroenterology

Research and Practice
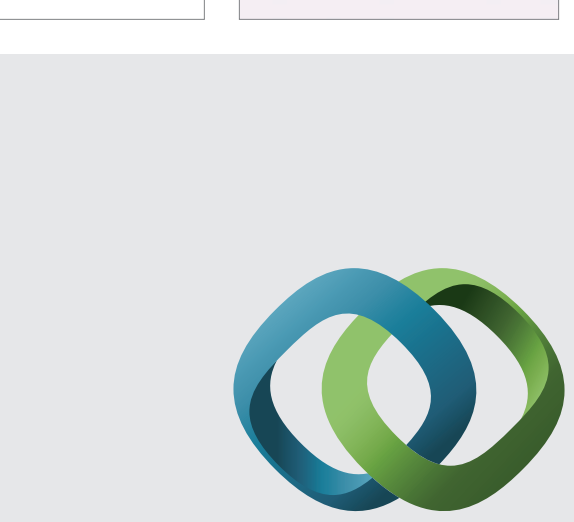

\section{Hindawi}

Submit your manuscripts at

http://www.hindawi.com
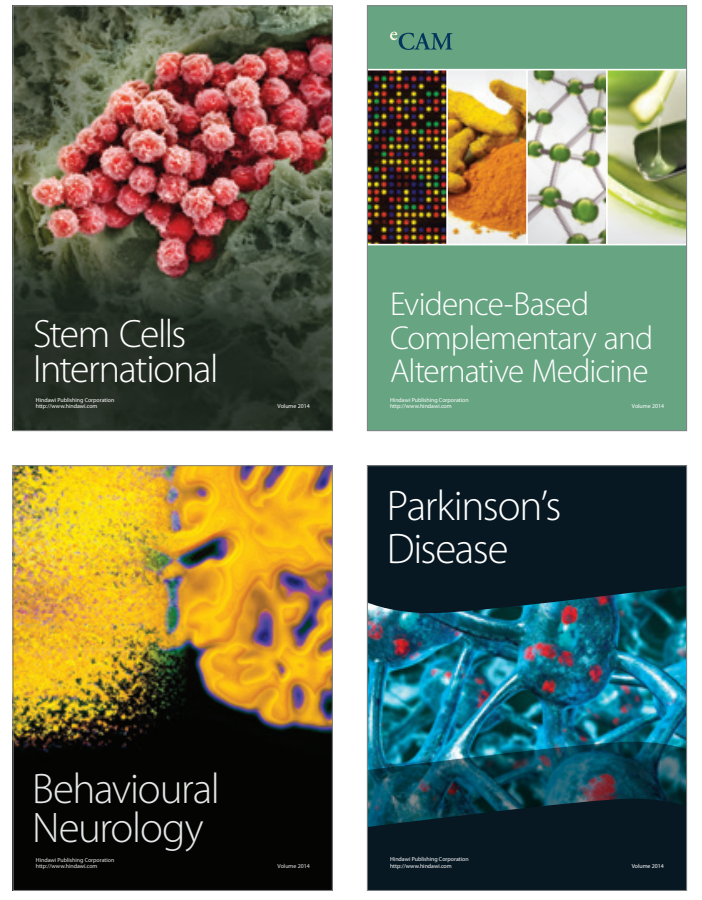
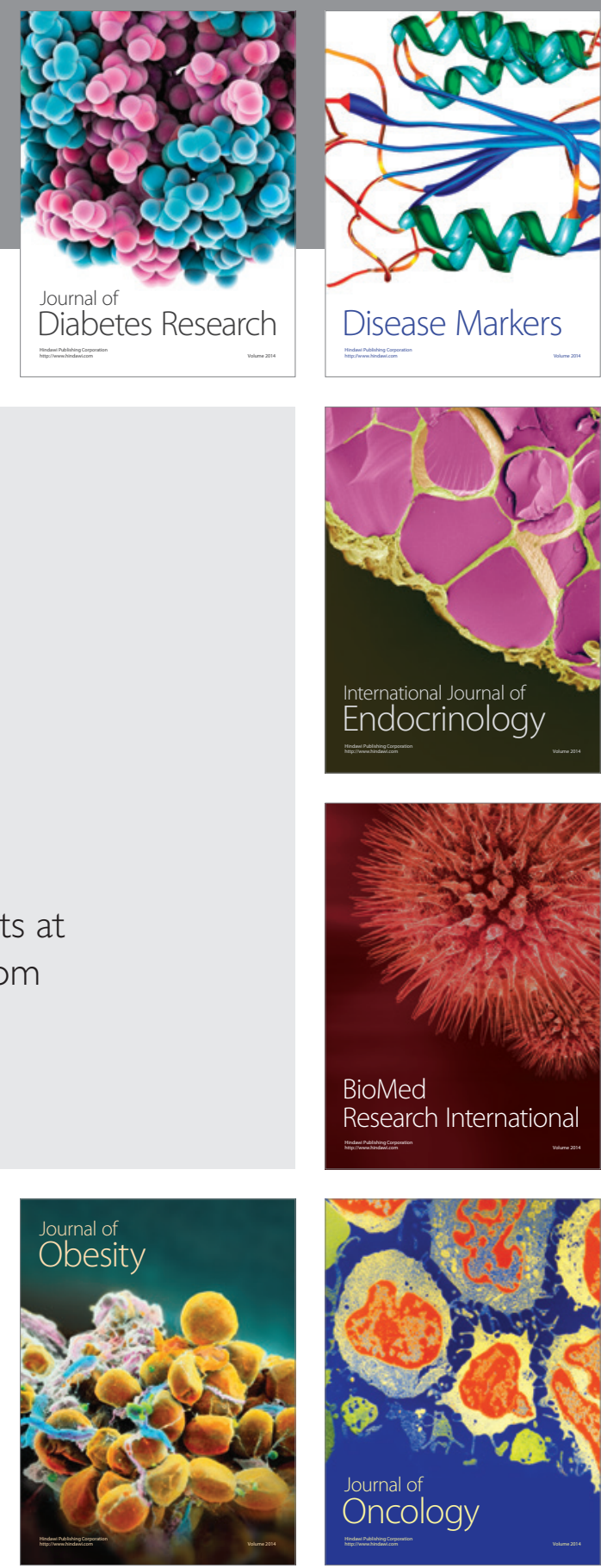

Disease Markers
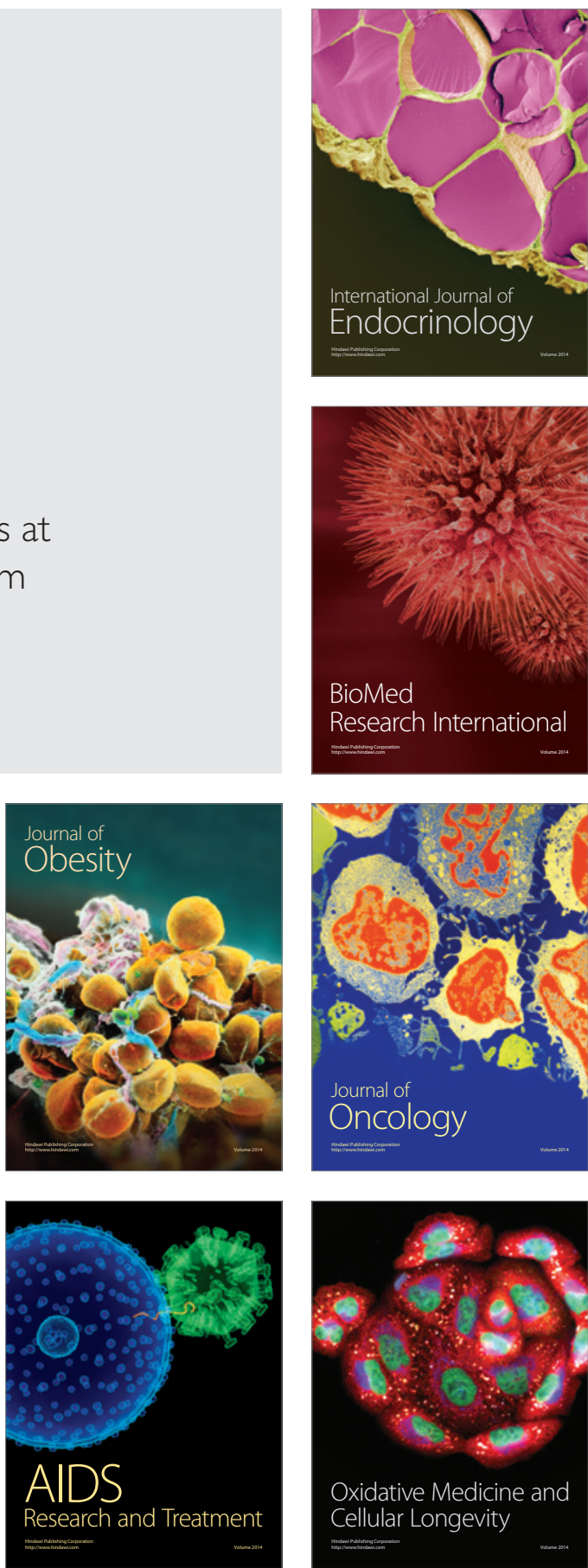